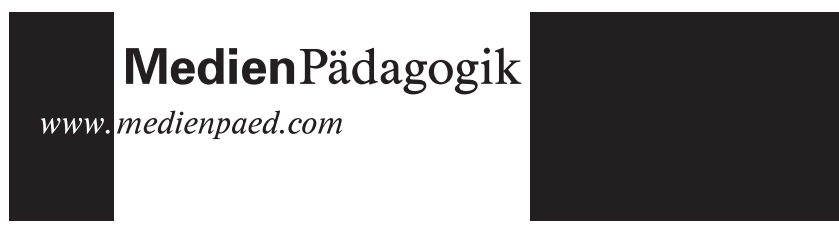

Ina Siekmann

28.5.2001

\section{Emotionalität in Internet-Seminaren}

«Internet-Seminare? Seminare im Internet?» «Ein Internet-Seminar ... wie sollen denn da richtige Diskussionen stattfinden? Da fehlt doch die Seminar-Stimmung! $\mathrm{Zu}$ meiner Studentenzeit wurde noch heftig von Angesicht zu Angesicht diskutiert!» «Vereinsamen die Studierenden nicht, wenn sie so allein vor ihrem Computer sitzen?» ...

Von wegen!

Mit solchen oder ähnlichen Bemerkungen wurde ich während der Zeit des ersten Internet-Seminars häufig konfrontiert. Da meine Erfahrungen zur Emotionalität in Internet-Seminaren andere sind als die oben geschilderten Vorurteile, möchte ich von meinen Erfahrungen als Tutorin eines Internet-Seminars berichten.

Über Aufbau und Ablauf eines solchen Internet-Seminares schreibt Herr Horst Heidbrink in dieser Ausgabe, so dass ich nur Besonderheiten hervorheben werde.

Das Seminar fand im Wintersemester 1997/98 im Lehrgebiet «Theorie der Schule und des Unterrichts» der FernUniversität Hagen statt. Es wurde von einem Moderator und von mir als Tutorin geleitet. An dem Seminar nahmen Studierende teil, die grösstenteils aus Deutschland, aber auch aus Österreich, Italien und Kolumbien kamen.

Anders als bei den Seminaren von Herrn Heidbrink wollten wir entsprechend einem gemässigten konstruktivistischen Lernstilverständnis von Anfang an wenig in das Geschehen eingreifen. Es sollten nur Aufgaben und Ziele vorgegeben und bei Bedarf Hilfestellungen und sonstige Tipps gegeben werden. Auf diese Weise sollte vermieden werden, dass die Teilnehmer/innen das Wissen der Moderatoren durch deren geschickte Lenkung «nachkonstruieren». Vielmehr sollten die Arbeitsergebnisse Ausdruck der Konstruktion von Wissen sein, das in Diskussionen über das jeweilige Thema erlangt wurde.

Die Seminarleitung gab am Beginn des Seminars einige Hinweise zu themenrelevanten Internetseiten (die Literatur sollte möglichst aus dem Internet bezogen werden), allgemein nützliche Internetadressen sowie Adressen nützlicher Suchmaschinen. Auch erfolgten allgemeine Tipps zum Umgang mit dem Internet, um besonders Internet-Anfängern den Einstieg zu erleichtern und sie vor einem «lost in cyberspace» zu bewahren.

Für die Studierenden bedeutete die offene Seminargestaltung, dass in ihren AGs alle weiteren Vorgehensweisen abgesprochen werden mussten: Wie wird weiter vorgegangen? Wer übernimmt welche Aufgabe? Wie oft meldet sich jeder zu Wort? Arbeitet jeder nach vorheriger Absprache für sich oder arbeitet man an Referatsteilen zusammen? Wie sieht die Zeiteinteilung aus? Wann müssen die einzelnen Referatsteile fertig sein, damit noch genügend Zeit zum Diskutieren bleibt? Wer übernimmt das Zusammenschreiben des Referates aus den einzelnen Stücken? usw.

Diese Vorgehensweise entspricht in etwa dem Arbeiten bei Gruppenarbeit in Präsenzveranstaltungen.

Für das Seminar waren keine online-Sitzungen (Chats) vorgesehen. Denn im Internet-Seminar soll ja gerade der Vorteil ausgenutzt werden, zeitunabhängig zu kommunizieren. Die AGs haben sich jedoch «privat» zu Online-Diskussionen getroffen. Ihnen ging es zum einen darum, auch einmal die Erfahrung zu machen, wie das zeitgleiche Chatten so geht, aber auch darum, spontane Fragen zu klären und die Arbeit etwas persönlicher zu gestalten.

Eindrücke und Indizien zur Emotionalität in Internet-Seminaren Da unsere Internet-Seminare in Newsgroups ${ }^{1}$ stattfanden und damit textbasiert waren, fehlten non-verbale Kommunikationsmöglichkeiten. Das kann damit aufgefangen werden, in dem man sehr ausführlich schreibt, Füllwörter benutzt und den Text an bestimmten Stellen mit Emoticons versieht. Aber genau das widerstrebt oft Internetnutzern, denn gerade das Schreiben von E-Mails unterstützt kurze, knappe, oft auch unvollständige

\footnotetext{
${ }^{1}$ Newsgroups sind virtuelle (in unserem Fall frei zugängliche) «Seminarräume», in denen Nachrichten hinterlassen werden können. Sie lassen sich vergleichen mit so genannten «Schwarzen Brettern», an die Anfragen angesteckt und von allen Anwesenden gelesen und beantwortet werden können (z.B. zum Thema «Zimmersuche» oder «Mitfahrgelegenheiten»).
} 
Sätze. Aber gerade diese kurzen, knappen Sätze sind es, die bei fehlender non-verbaler Kommunikation sehr leicht missverstanden werden können. Kurze, knappe Sätze erinnern schnell an Befehle, und wenn das freundliche Gesicht fehlt, mit dessen Hilfe das Gesagte besser einzuschätzen ist, können sich einige Teilnehmer/innen schnell von solchen Sätzen verunsichert oder sogar eingeschüchtert fühlen.

Die Bemerkung einer Teilnehmerin passt hierzu: «Ich finde, das Arbeiten in Newsgroups fördert einen Kommunikationsstil, der eher kurz, prägnant und wenig einfühlsam ist. Man neigt dazu, Meinungen $\mathrm{zu}$ formulieren, die man im persönlichen Umgang vielleicht so nicht sagen würde oder zumindest abschwächen kann. ... sollten wir etwas vorsichtiger in der Formulierung und etwas weniger mimosenhaft in der Wahrnehmung werden.» (AG 2, TN F², 14. Dezember 1997)

Eine weitere Auffälligkeit, die ich in mehreren Seminaren beobachtet habe, kann auf das Fehlen von non-verbaler Kommunikation zurückgeführt werden: Wenn die Teilnehmer/innen an einem Beitrag Kritik üben wollten, dann begannen sie zuerst mit etwas Positivem, bevor ihre Kritik folgte; sie versuchten, niemanden auf die Füsse zu treten. Auch hier fehlt das freundliche Gesicht, die aufmunternde Stimme des Gegenüber, die signalisieren, dass man trotz Kritik dem- oder derjenigen noch freundlich gesinnt ist.

Internet-Seminare stossen auch auf technische Schwierigkeiten: Während es relativ einfach ist, jemanden in einer Präsensveranstaltung zu erklären, wie man bspw. zu einer bestimmten Suchmaschine im Internet gelangt, in dem man dem- oder derjenigen einfach auf dem Bildschirm zeigt, wohin er/sie mit welcher Maustaste klicken soll, wird dies in Internet-Seminaren durch die Nichtpräsenz der Studierenden erschwert. Das einfache Mausklicken, das Eingeben von Suchbegriffen an der richtigen Stelle, das Installieren von Programmen usw. leicht verständlich zu beschreiben, ist viel mehr Aufwand. Daneben fehlt auch die unmittelbare Rückmeldung, ob der- oder diejenige die Erklärung verstanden hat.

Aber trotz dieser möglichen Schwierigkeiten bin ich überzeugt, dass sich Internet-Seminare und Emotionaltität nicht ausschliessen, sondern sich gut miteinander verbinden lassen. Das möchte ich anhand einiger Beispiele verdeutlichen.

2 Abkürzung aus Datenschutzgründen anstelle des Namens

\section{Emotionale Textbeispiele aus der Praxis}

Die folgenden Beispiele sind einem Internet-Seminar entnommen, das im Wintersemester 1997/98 am Lehrgebiet «Theorie der Schule und des Unterrichts» an der FernUniversität Hagen zum Thema «Schule und Neue Medien» stattgefunden hat. Ich stelle die Textbeispiele ausführlich dar, da sie die von mir aufgestellten Behauptungen eindrucksvoll belegen. Ich unterscheide dabei positive und negative Äusserungen.

\section{Virtuelles Schulterklopfen: Positive Äusserungen}

«Wunderbar, von Dir zu hören, dass Du mitmachst. ... Auf jeden Fall ist es schön, dass Du nun gesund bist und mit mir gemeinsam weiterwurschteln kannst. Gestern war ich am Boden zerstört, meinen Wutschrei hast Du gehört. War vielleicht ein wenig heftig, aber ich ärgere mich über so wenig Solidarität! Auf gute Zusammenarbeit! :-) » (TN A, 23.12.97)

« ... ich habe gerade Dein Referat gelesen. Super! Passt genau zu dem, was jetzt von mir kommt, bilde ich mir ein. ...» (TN A, 16.12.97)

«... ich glaube, wir können uns weiter in unserer AG unterhalten, da wir ja doch nur noch zu zweit sind. Inzwischen bist Du mir schon so vertraut geworden, dass ich immer neugierig nachschaue, ob schon eine Anwort da ist. Und dabei gibt es Leute, die behaupten, Computer machen einsam!» (TN B, 17.12.97)

«... ich wollte ... Euch virtuell auf die Schulter klopfen. Ihr seid ja wahnsinnig fleissig, die News in Eurer AG sind fast unzählig. Toll, dass Ihr so gut zusammenarbeitet, wo die anderen sich etwas <zurückgezogen〉 haben. Also, wirklich Hut ab!!! ...» (TN C, 23.12.97)

\section{Negative Äusserungen}

«Bevor ich an meinem Ärger ersticke, muss ich meinem Herzen doch mal Luft machen. Liebe Leute, was ist das für eine Gruppe, in der ich da gelandet bin? Die erste Kommilitonin hat sich gleich zu Anfang verabschiedet, die zweite taucht gar nicht erst auf, zwei Kommilitoninnen versinken im Motivationstief und nur eine meldet sich zaghaft! Zum Donnerwetter, Selbstgespräche kann ich auch zu Hause führen, dazu brauche ich keinen Computer. ... Zwischenprüfung und Fortbildung lasse ich gelten, fehlende Motivation und Power aber nicht. Mit Verlaub, ich sag's ganz deutlich: ich fühle mich total verarscht. Stinksauer aus dem Odenwald! :-(((» (TN A, 12.12.97) 
«... So intensiv, wie Ihr beiden das betreibt, muss man es sicher nicht tun. Das ist gefährlich, weil es schnell in Überforderung enden kann. Ich könnte mir diese Arbeitsweise nur zeitweise leisten. Die übrige Zeit muss ich etwas für Butterbrote tun. Da bestimmen andere meine Zeit. ... Eue Tempo halte ich persönlich auch gar nicht für so erstrebenswert. Auch die Gesprächsmenge wäre mir zuviel. Reden soll man immer nur dann, wenn es Unklarheiten gibt. Wenn man weiss, was man tut, muss man nicht pausenlos darüber debattieren. (TN D, 14. Januar 1998)

«Deine Antwort auf 〈TN As〉 Frage über die Erwartungen habe ich als Vorwurf empfunden, als ob wir das wissenschaftliche Forum als Quatschecke benutzen würden. Ich weiss, du hast es nicht genauso ausgedrückt. Doch schwang zwischen den Zeilen diese Unterstellung mit. Wer lässt sich so etwas schon gerne schreiben. Wir selbst sind stolz auf unseren guten Austausch und von der Ernsthaftigkeit und Zielstrebigkeit genauso überzeugt wie du von deiner Arbeit.» ... Unseren Meinungsaustausch empfinden wir nicht als anonyme Abfrage, da $\langle\mathrm{TN}$ A $>$ und ich uns durch unseren intensiven Austausch inzwischen so vertraut sind, dass das Wort anonym hier fehl am Platz ist. Wir haben die Erfahrung gemacht, dass wir auch im virtuellen Raum ohne reale Begegnung menschliche soziale Kontakte aufbauen können und Tugenden wie Verlässlichkeit, Zuverlässigkeit, Ehrlichkeit, Humor, Flexibilität, Vertrauen und das Aufeinander-Einlassen-Können finden.» (TN B, 23. Januar 1998)

«... es wird Zeit, dass ich direkt Stellung nehme zu dem, was Du schreibst, nicht hintenherum und indirekt, sondern dir sehr deutlich sage, was ich denke und empfinde. Meiner Meinung nach hat $\langle$ TN B $\rangle$ Dir sehr freundlich und einlenkend geantwortet, und ich habe das in meiner ersten Antwort im Café auch versucht. Offensichtlich verstehst Du diese Sprache nicht. Du fühlst Dich auf den Schlips getreten, warum? Lies Dir in aller Ruhe durch, was Du auf meine Anfrage geantwortet hast. Meiner Meinung nach kann man das nur als massive und schlecht getarnte Vorwürfe begreifen. ... Jedoch habe ich nicht im Traum daran gedacht, dass sich ein Grossteil einer persönlichen Antwort in Vorwürfen erstrecken könnte.

Was soll das? Es ist schlicht eine Unverschämtheit, unser Tempo als nicht erstrebenswert und unsere Gesprächsmenge als zuviel zu bezeichnen, noch dazu, nachdem Du Dir kurz zuvor nicht verkneifen konntest, auf Deine Berufstätigkeit zu verweisen! Ich würde Dir gerne darlegen, wie ich Deine Darstellung lese: hart arbeitender Mann, der für die Brötchen der
Familie sorgt und nebenbei zielstrebig studiert. Dagegen diese Hausfrauen mit ihrem Geschwätz, die sich über Nichtigkeiten austauschen und alle Zeit der Welt haben. Ich werde den Teufel tun und Dir erzählen, was ich neben dem Studium noch alles mache, aber sei gewiss, vom Arbeitspensum her kann ich allemal mithalten. ... Wenn wir uns mitteilen, Akte $\mathrm{x}$ von Autor y gefunden zu haben, dann heisst das nicht: guck mal, was ich gefunden habe, ätsch, sondern soll ein Lesehinweis für die Partnerin sein, damit man über den jeweiligen Text sprechen kann. ... Und nun wünsche ich uns allen eine friedliche Koexistenz, vielleicht sogar Zusammenarbeit.» (TN A, 23. Januar 1998)

«Bei Dir, 〈TN A〉, vermisse ich ein bisschen von einer anderen Tugend der Freundlichkeit. Ob ich 〈offensichtlich〉 eine Sprache nicht verstehe, wollen wir mal dahingestellt sein lassen. Ich glaube nicht, dass Du das beurteilen kannst. Im übrigen erbitte ich zukünftig keine persönliche Einmischungen in meine Gespräche mit meiner Gruppenpartnerin. ...

Nun lasst uns aber nicht zanken. ... Dass ihr beiden nicht angegriffen wurdet, habt ihr schliesslich selbst erkannt. Wenn ihr Euch trotzdem auf Ungesagtes 〈zwischen den Zeilen〉 einlasst, gibt es dagegen keine Chance für eine Rechtfertigung. ... Mein Hinweis auf meine Berufstätigkeit ... (Warum soll hierin ein 〈Hintergedanke〉 oder eine 〈böse Absicht〉 enthalten sein? Wo liegt hier ein massiver und schlecht getarnter Vorwurf?)» (TN D, 24. Januar 1998)

Nicht nur die Teilnehmer/innen empfanden das Streitgespräch zwischen den beiden Frauen und dem einen Mann als unangenehm und wollten es schlichten, auch die Seminarleitung war um ein Beenden bemüht:

«Liebe Leute, vielleicht liegt es ja daran, dass man beim Schreiben die Worte anders setzt als bei der direkten Kommunikation mit einem Gegenüber, den man sieht... Aus diesem Seminar gewinnen wie alle am meisten, wenn wir zumindest zusammenarbeiten und jeden akzeptieren, wie er (oder sie) ist, die Arbeitsstile differieren naturgemäss nach Zeitbudget, und man muss ja nicht alles genauso machen wie die anderen.» (TN E, 28. Januar 1998

«Also, ich möchte nicht, dass diese Diskussion in dieser AG weitergeführt wird. Sie gehört in die Café-Gruppe ... Die AGs sind keine Selbsterfahrungsgruppen. ... Dabei bitte ich die Beiträge so zu formulieren, dass sich niemand persönlich verletzt fühlt.» (Moderator, 23. Januar 1998) 


\section{Emoticons und Grussformeln}

Die Stimmungen der Studierenden des Seminars werden häufig durch die Verwendung von Emoticons und Grussformeln am Ende einer Mails angedeutet. Die folgenden Beispiele stammen von Mails einer Teilnehmerin (TN A) und machen den Effekt sehr deutlich: Dem Leser/die Leserin wird in die Stimmung der Absenderin vermittelt.

:-((( stinksauer aus dem Odenwald

:-) herzliche Grüsse, auf gute Zusammenarbeit, ...

:-)) schwungvoll aus dem Odenwald

:-)) Bis bald

:-() herzliche Grüsse und einen schönen dritten Advent wünscht

:-o total verschusselt aus dem Odenwald

ohne Emoticons:

müde aus dem Odenwald

erwartungsvoll aus dem eisigen Odenwald

verschneite Grüsse aus dem Odenwald

aus dem total verregneten Odenwald

\section{Zusammenfassung}

Meine Erfahrungen haben gezeigt, dass es durchaus mehrere Ebenen des Ausdrucks von Emotionen in Internet-Seminaren gibt: die Sprache selbst, bestimmte Formeln, bekannte und erfundene Symbole. Die Emotionalität in Internet-Seminaren ist stark an die Kommunikation gekoppelt. Um Emotionen in Schriftform überhaupt sichtbar zu machen, müssen sie geschrieben werden. So müssen statt eines verärgerten Schnaufens in einer Präsenzveranstaltung 2-3 Sätze geschrieben werden, die den Zustand der Teilnehmerin oder des Teilnehmers beschreiben und erklären. Wie einfach lassen sich doch Augenbrauen hochziehen, die das Anzweifeln eines Argumentes anzeigen sollen, aber wie sorgfältig müssen die Worte gewählt sein, um diese Empfindung auszudrücken!! Denn die Worte stehen bis zum Ende eines Seminars in der Newsgroup und können durch die Öffentlichkeit von allen gelesen werden, während verbale und nonverbale Kommunikation in Präsenzveranstaltungen nach einiger Zeit in Vergessenheit geraten. Wer also emotional ist (oder sein will), muss meiner Meinung nach auch kommunikativ sein.

Zum guten Schluss noch eine Anmerkung: Ich habe später erfahren, dass zwei Studentinnen, die sich im Internet-Seminar kennengelernt haben, quer durch Deutschland gefahren sind, um zusammen mit ihren Familien Ostern zu feiern!

«... das Internet ist doch so anonym und unterstützt Vereinsamung!» Von wegen!

Die Ergebnisse des Internet-Seminars sind nachzulesen unter: 〈http://www.fernuni-hagen.de/ERZBIL/TDS/agvir/ergebnisse.htm〉 (Stand: 29.03.2001) 